\title{
Large time convergence in Hamilton-Jacobi equations
}

\section{Jean-Michel Roquejoffre*}

Laboratoire MIP-CNRS and Institut Universitaire de France

Université Paul Sabatier, 118 route de Narbonne, 31062 Toulouse Cedex, France

E-mail: roque@mip.ups-tlse.fn

Large time behaviour problems in Hamilton-Jacobi equations have recently attracted a lot of interest, due to their links to Hamiltonian dynamics, but also to physical applications (flame propagation, homogenisation). This paper is an attempt to provide the current state of the art on this question.

Control Systems: Theory, Numerics and Applications

30 March - 1 April 2005

Rome

${ }^{*}$ Speaker. 


\section{Introduction}

Consider the Cauchy Problem

$$
\begin{gathered}
u_{t}+H(x, D u)=0 \quad \text { in }(0,+\infty) \times \mathbb{R}^{n}, \\
u(x, 0)=u_{0}(x) \quad \text { in } \mathbb{R}^{n} .
\end{gathered}
$$

Typically, $H \in C\left(\mathbb{R}^{n} \times \mathbb{R}^{n}\right)$ and $u_{0} \in W^{1, \infty}\left(\mathbb{R}^{n}\right)$ are assumed to be periodic in $x$, while $H(x, p)$ is also assumed to be convex, smooth and coercive in p, i.e.

$$
H(x, p) \rightarrow+\infty \quad \text { as }|p| \rightarrow+\infty \quad \text { uniformly w.r.t. } x \in \mathbb{R}^{n} .
$$

As a consequence of these assumptions, the solutions are global in time, Lipschitz continuous and periodic in $x$. See [3] for a very complete account of what can be expected.

The first step in the study of large time behaviour in (1.1-1.2 consists in solving a so-called ergodic problem, namely a stationary Hamilton-Jacobi Equation of the type

$$
H(x, D \phi)=\lambda \quad \text { in } \mathbb{R}^{n} .
$$

where both the function $\phi$ and the constant $\lambda$ are unknown. The key result here is due to LionsPapanicolaou-Varadhan [25], and was intended to homogenisation: there exists a unique constant $\lambda$ such that (1.4) has a Lipschitz continous, periodic solution. It is worth remarking at this stage that the actual interest of this result is to provide a bounded solution and this is where periodicity plays a key role. This property breaks down when periodicity is dropped, and this becomes a major difficulty. See for instance [12] for homogenisation results in nonlinear random media.

The connection with large time behaviour is then as follows: one first proves that the solution $u$ of (1.1)-(1.2) satisfies

$$
\frac{u(t, x)}{t} \rightarrow-\lambda \quad \text { as } t \rightarrow+\infty \quad \text { uniformly in } \mathbb{R}^{n},
$$

then one may examine whether a property of the form

$$
u(t, x)+\lambda t \rightarrow u_{\infty}(x) \quad \text { as } t \rightarrow+\infty, \quad \text { uniformly in } \mathbb{R}^{n},
$$

where $u_{\infty}$ is a solution of (1.4), is true. It is worth pointing out that, if a property like (1.5) can be obtained rather easily as a consequence of the standard maximum principle for (1.1), the more precise asymptotic behavior (1.6) is, on the contrary, a far more difficult result ; in fact, the asymptotic behavior of solutions of (1.1)-(1.2) remained an open problem for a long time - all the more as (1.6) may not be true.

This might explain why large time behaviour received very little attention before the last 8 to 10 years. Namah and Roquejoffre [26] were the first to break the difficulty under the following additional assumptions

$$
H(x, p) \geq H(x, 0) \quad \text { in } \mathbb{R}^{n} \times \mathbb{R}^{n} \quad \text { and } \quad \max _{\mathbb{R}^{n}} H(x, 0)=0 .
$$

This assumption, which can be slightly generalized by replacing $H(x, 0)$ by $H(x, D \varphi(x))$ where $\varphi: \mathbb{R}^{n} \rightarrow \mathbb{R}$ is a $C^{1}$, Lipschitz continuous function, seems to be a bit restrictive. However, it 
covers several interesting cases - such as reversible hamiltonians: $H(x, p)=H(x,-p)$ and, on the other hand, does not require strict convexity assumptions on $H$ in $p$.

An important general result is due to Fathi [18], who proved (1.6) with dynamical systems type arguments, and in particular giving to the Aubry-Mather set - roughly speaking, an attractor for the geodesics associated to the representation formula of $u$ - a central role. Contrarily to Namah and Roquejoffre, Fathi uses strict convexity assumptions on $H$ (and also far more regularity) but he does not need (1.7) and his proof can indeed handle cases where (1.7) is not satisfied. In fact, (1.7) can be interpreted as a case where the Mather set has a particular form. Fathi's proof was revisited by Roquejoffre, who managed to drop the use of the conservation of the Hamiltonian along the geodesics. This allowed him to prove partial results in the 1D time dependent case [29]. This result was completed by Bernard [9], and a much simplified proof, relying on some ideas in [29], was issued in [10].

A paper of Barles and Souganidis [7], which generalizes both Namah-Roquejoffre and Fathi's results and which can even handle some cases where $H$ is not convex in $p$. The key assumption is on the quantity $H_{p}(x, p) \cdot p-H(x, p)$ which, in some sense, measures the attractivity of the Mather set. A dynamical systems approach is given for less smooth hamiltonians - essentially keeping only the strict convexity - in an interesting preprint of Davini and Siconolfi [14].

Although the question can be considered as settled in the time independent, space periodic case, important open problems remain, especially when the hamiltonian $H$ is allowed to depend on time, for instance in a periodic fashion. We will also see that (1.6) is not be true in certain cases. The paper is organised as follows: first, we shall give the main lines of a proof for 1.6 in the periodic, strictly convex case; this will be the object of Section 2. Then we will examine where the proof breaks down, and give an account of some cases when it can be mended, and some cases hen the result is simply not true: to this end we will examine the time-periodic and non strictly convex cases in Section 3, and the non spatially periodic case in Section 4.

Acknowledgement. This paper is based on a talk that I was invited to give at the INDAM conference 'Control and Numerics', held by Profs. Cannarsa, Capuzzo-Dolcetta, Garavello and Piccoli. It is my pleasure to thank them for this very interesting meeting, and for their hospitality.

\section{Convergence in the smooth, spatially periodic, strictly convex case}

Let $\mathbf{T}^{n}$ be the $n$-dimensional unit torus. The main result of this section is the following.

Theorem 1. ([[18], [29], [/]]) Assume the hamiltonian $H(x, p)$ to be smooth, 1-periodic in $x$, superlinear and strictly convex in $p$ - uniformly with respect to $x$. Let $u_{0}(x)$ be a continuous, 1-periodic Cauchy datum. Then there is a solution $\phi(x)$ of (1.4) such that the solution $u(t, x)$ of $(1.1-1.2)$ satisfies

$$
\lim _{t \rightarrow+\infty}(u(t, x)-\phi)=0 .
$$

The general scheme is as follows: first, one identifies a uniqueness set for (1.4), i.e. a closed subset of $\mathbf{T}^{n}$, called $\mathcal{M}_{0}$, such that, if $\lambda$ is the only constant for which (1.4) has solutions, then the Dirichlet problem

$$
H(x, D u)=0 \text { in } \mathbf{T}^{n} \backslash \mathcal{M}_{0}, \quad u \text { imposed on } \mathcal{M}_{0}
$$


has at most one solution. It is the main contribution of Fathi [17], [18] to have identified such a class of sets - Aubry-Mather sets. Once this is done, the main task is to prove that the solution of the Cauchy Problem converges, on $\mathcal{M}_{0}$, to a solution of (1.4). We then conclude by a Barles-Perthame type argument.

From now on the constant $\lambda$ in (1.4) will be, without loss of generality, set to 0 .

\subsection{General properties of viscosity solutions}

We recall in this section some well understood properties of viscosity solutions without proof, see [24], [3], or [17]. Let us denote $\mathcal{T}(t): C\left(\mathbf{T}^{n}, \mathbb{R}\right) \longrightarrow C\left(\mathbf{T}^{n}, \mathbb{R}\right)$ the mapping which associates to each function $u_{0} \in C\left(\mathbf{T}^{n}, \mathbb{R}\right)$ the function $u(t,$.$) , where u(t, x) \in C\left(\left[0,+\infty\left[\times \mathbf{T}^{n}, \mathbb{R}\right)\right.\right.$ is the viscosity solution of (3.1) such that $u(0,)=.u_{0}$.

We have the semigroup property

$$
\mathcal{T}\left(t^{\prime}-t\right) \mathcal{T}(t)=\mathcal{T}\left(t^{\prime}\right)
$$

for $0 \leq t \leq t^{\prime}$. Moreover the mappings $\mathcal{T}(t)$ are contractions,

$$
\|\mathcal{T}(t) u-\mathcal{T}(t) v\|_{\infty} \leq\|u-v\|_{\infty} .
$$

Finally, the mappings $\mathcal{T}(t)$ are compact. More precisely, given a bounded set $B \subset C\left(\mathbf{T}^{n}, \mathbb{R}\right)$, there exists a positive nondecreasing function $K(\varepsilon):] 0, \infty[\longrightarrow] 0, \infty[$ such that $\mathcal{T}(t) u$ is $K(\varepsilon)$-Lipschitz for all $u \in B$ and all $t \geq s+\varepsilon$. See [24]

The mappings $\mathcal{T}(t)$ are nondecreasing and satisfy $\mathcal{T}(t)(u+c)=c+\mathcal{T}(t)(u)$ for all real $c$.

\subsection{Lax-Oleinik formula, calibrated curves and Aubry-Mather set}

In this section, we recall some consequences of the "explicit" Lax-Oleinik formula, culminating with the construction of the Aubry-Mather set as a uniqueness set for (1.4). Almost no proofs will be given here; we refer to [17], [18], [29] for the proofs.

The Lax-Oleinik formula is

$$
\mathcal{T}(t) u_{0}=\inf _{\gamma}\left(u_{0}(\gamma(0))+\int_{0}^{t} L(\gamma, \dot{\gamma}) d \sigma\right)
$$

where the infimum is taken on the set of piecewise $C^{1}$ curves with values in $\mathbf{T}^{n}$, such that $\gamma(t)=x$. In the above, the Lagrangian $L(x, v)$ is defined as the Legendre transform of $H$ :

$$
L(x, v)=\max _{p \in \mathbb{R}}(p \cdot v-H(x, p)) .
$$

It is a classical result from the calculus of variations that, under our assumptions, the infimum in (2.2) is reached by $C^{2}$ curves. The Lipschitz constant of these curves only depends on $L$.

Let $u:\left[0,+\infty\left[\times \mathbf{T}^{n} \longrightarrow \mathbb{R}\right.\right.$ be a viscosity solution of (1.1). A curve $\gamma:\left[0,+\infty\left[\supset\left[t, t^{\prime}\right] \longrightarrow \mathbf{T}^{n}\right.\right.$ is said calibrated by $u$ on the interval $\left(t, t^{\prime}\right)$ if

$$
u\left(t^{\prime}, \gamma\left(t^{\prime}\right)\right)=u(t, \gamma(t))+\int_{t}^{t^{\prime}} L(\gamma, \dot{\gamma}) d \sigma
$$


From [17], if $\gamma:\left[t, t^{\prime}\right] \longrightarrow \mathbf{T}^{n}$ is calibrated by $u$, then $u_{x}$ exists at each point $(s, \gamma(s)), s \in\left[t, t^{\prime}[\right.$ and satisfies

$$
u_{x}(s, \gamma(s))=L_{v}(\gamma(s), \dot{\gamma}(s)) \Longleftrightarrow \dot{\gamma}(s)=H_{p}\left(\gamma(s), u_{x}(s, \gamma(s)) .\right.
$$

More precisely, there exists a positive nondecreasing function $K(\varepsilon):] 0, \infty[\longrightarrow] 0, \infty[$ such that any minimizing curve $\gamma:[t, t+\varepsilon] \longrightarrow \mathbf{T}^{n}$ satisfies $\|(\dot{\gamma}, \ddot{\gamma})\|_{\infty} \leq K(\varepsilon)$.

Such a result was already lurking in [28], but was not made that systematic.

Let us now consider a solution $\phi(x)$ of (1.4). The Lax-Oleinik formula applies to $\phi$, and a classical compactness argument gives the existence of curves $\gamma: \mathbb{R} \longrightarrow \mathbf{T}^{n}$ which are calibrated by $\phi$ on all compact interval.

It is a consequence of [17], or Mather's shortening lemma, see [23] that two such curves cannnot intersect. More precisely if $\gamma_{1}$ and $\gamma_{2}: \mathbb{R} \longrightarrow \mathbf{T}^{n}$ are calibrated by $\phi$, and if there exists a $t$ such that $\gamma_{1}(t)=\gamma_{2}(t)$, then $\gamma_{1}=\gamma_{2}$. It interested to see that some forms of this non-intersection propery holds for higher dimensional objects, such as hypersurfaces; see a spectacular example in Caffarelli-de la Llave [11].

Let $\mathcal{D}_{\phi} \subset \mathbf{T}^{n}$ be the set of points $\gamma(0)$, where $\gamma: \mathbb{R} \longrightarrow \mathbf{T}^{n}$ is calibrated by $\phi$ on the whole interval $(-\infty,+\infty)$. This is a nonempty compact set. For each $t$, we define the mapping $S_{\phi}^{t}: \mathcal{D} \longrightarrow$ $\mathbf{T}^{n}$ which associates to each $x \in \mathcal{D}_{\phi}$, the value $\gamma(t)$, where $\gamma: \mathbb{R} \longrightarrow \mathbf{T}^{n}$ is the unique calibrated curve satisfying $\gamma(0)=x$. It is a bi-Lipschitz homeomorphism onto its image. Let us note $\mathcal{M}_{\phi}$ its global attractor: this is the closure in $\mathbf{T}^{n}$ the set of points $x \in \mathcal{D}_{\phi}$ which are the limit of a sequence $S^{t_{k}}(y)$ with $y \in \mathcal{D}_{\phi}$ and $\left(t_{k}\right)_{k}$ an indefinitely increasing sequence. The set $\mathcal{M}_{\phi}$ is non-empty and compact. We call $\mathcal{M}$ the union, in $\mathbb{R} \times \mathbf{T}^{n}$, of the graphs of curves $S_{\phi}^{t}(x), x \in \mathcal{M}_{\phi}$.

All the objects constructed so far: the sets $\mathcal{D}_{\phi}$ and $\mathcal{M}_{\phi}$, as well as the mapping $S_{\phi}^{t}$, depend on the steady solution $\phi$ that was chosen in the beginning. Let us therefore define the sets

$$
\mathcal{A}_{0}=\bigcap_{\phi} \mathcal{D}_{\phi}, \quad \mathcal{M}_{0}=\bigcap_{\phi} \mathcal{D}_{\phi},
$$

where the intersection is taken on the set of steady solutions. This last set is usually called the Aubry-Mather set. We will see in the next paragraph that $\mathcal{M}_{0}$ is non void, and will play the uniqueness role that we expect.

Example. Assume that the hamiltonian $H(x, p)$ has the form $|p|^{2}-f(x)$, where $f$ is a smooth, nonnegative function. Then $\mathcal{M}_{0}$ is exactly the zero set $Z$ of $f$ : indeed, we have

$$
L(x, v)=\frac{1}{4}|v|^{2}+f(x) .
$$

It is then easily seen from the Lax-Oleinik formula that a curve $\gamma$, calibrated by $\phi$ on $\mathbb{R}$, should have

$$
\lim _{t \rightarrow+\infty} f(\gamma(t))=0:
$$

if not, then we have - taking into account that $|\dot{\gamma}|$ is bounded: $\lim _{t \rightarrow=\infty} \mathbf{T}(t) \phi(\gamma(0))=+\infty$. Hence $\mathcal{M}_{0} \subset Z$. Conversely, if $x_{0} \in Z$, we have $S_{\phi}^{t}\left(x_{0}\right)=x_{0}$.

One may prove directly, by a trivial adaptation of the uniqueness proof in [3], that $\mathcal{M}_{0}$ is a uniqueness set in this case. A complete study of the equation $|D u|^{2}-f(x)=0$ in a bounded open subset of $\mathbb{R}^{n}$ - the study in $\mathbf{T}^{n}$ is completely equivalent - is presented in [24]. 


\subsection{The Aubry-Mather set is a uniqueness set; convergence}

The following remark, made in [29], is the key point to the convergence proof:

Lemma 1. Let $u(t, x):\left[s, \infty\left[\times \mathbf{T}^{n} \longrightarrow \mathbb{R}\right.\right.$ be a viscosity solution of $\left.(1.1]\right)$, let $\phi(x)$ be a steady solution. Let $x \in \mathcal{D}_{\phi}$. Then the function $t \mapsto u\left(t, S_{\phi}^{t}(x)\right)-\phi\left(S_{\phi}^{t}(x)\right)$ is non-increasing.

The $\omega$-limit set of $u_{0}$ with respect to the semigroup $\mathcal{T}$ is then classically defined as

$$
\omega\left(u_{0}\right)=\left\{\psi \in C\left(T^{n}\right): \exists\left(t_{n}\right)_{n} \rightarrow+\infty: \lim _{n \rightarrow+\infty}\left\|\mathcal{T}\left(t_{n}\right)-\psi\right\|_{\infty}=0\right\} .
$$

From Section 2.1, $\omega\left(u_{0}\right)$ is a nonvoid compact, connected subset of $C\left(T^{n}\right)$.

The important consequence of Lemma 1 is also proved in [29]:

Corollary 1. Let $\psi$ be in $\omega\left(u_{0}\right)$, and choose $x \in \mathcal{M}_{\phi}$. Then the function $t \in \mathbb{R} \mapsto \mathcal{T}(t) \psi\left(S_{\phi}^{t}(x)\right)-$ $\phi\left(S_{\phi}^{t}(x)\right)$ is constant.

It follows that the curve $S_{\phi}^{t}(x)$ is calibrated by $\mathcal{T}(t) \psi$. But notice that this fact has nothing to do with the particular choice of $u_{0}$ : it just follows from the fact that $\mathcal{T}(t) \psi$ is a time-global solution of (1.1). The consequence is then the following, originally due to Fathi [17]:

Theorem 2. $\mathcal{M}_{0}$ is a uniqueness set.

With the aid of Corollary 1 , the proof is easy: let indeed $\phi_{1}$ and $\phi_{2}$ be two steady solutions of (1.1); assume that $\phi_{1}=\phi_{2}$ on $\mathcal{M}_{0}$. Take $x \in \mathbf{T}^{n}$ and consider

$$
y \in \omega_{S_{\phi_{1}}^{t}}(x), \quad z \in \omega_{S_{\phi_{1}}^{t}}(y)
$$

the subscript $S_{\phi_{1}}^{t}$ indicating that we are taking $\omega$-limit sets with respect to the dynamics of $S_{\phi_{1}}^{t}$. Corollary 11 implies that, if $\gamma(t)=S_{\phi_{1}}^{t}(y)$, then $\gamma$ is also calibrated by $\phi_{2}$. Lemma 1 implies that $\phi_{1}(y) \leq \phi_{2}(y)$, and the same argument with, this time, $S_{\phi_{2}}^{t}$ implies $\phi_{2}(y) \leq \phi_{1}(y)$. Hence the theorem.

Coming back to our $\omega$-limit solution $u(t,):.=\mathcal{T}(t) \psi$, we deduce that $u, \mathcal{T}(t) \varphi$ and $\phi$ are differentiable on $\mathcal{M}_{\phi}$. Consider $(t, x) \in \mathbb{R} \times \mathcal{M}_{0}$; a Taylor expansion of the equation

$$
\frac{d}{d t}(u-\phi)(t, x)=0
$$

at order 2 in $u-\phi$, together with the strict convexity of $H$, yields the

Corollary 2. For $(t, x) \in \mathbb{R} \times \mathcal{M}_{0}$ we have

$$
\partial_{t}(u-\phi)(t, x)=D(u-\phi)(t, x)=0 .
$$

This implies the convergence of $\mathcal{T}(t) u_{0}$ on $\mathcal{M}_{0}$.

Once convergence on $\mathcal{M}_{0}$ is secured, the convergence of the semigroup everywhere is granted by (by now) classical viscosity solutions arguments. 
Proposition 1. The function

$$
\phi(x)=\liminf _{t \rightarrow+\infty} \mathcal{T}(t) u_{0}(x)
$$

is a steady viscosity solution of (1.1).

It is a steady super-solution by the Barles-Perthame lemma [4]. That it is a sub-solution results from simple operations on the Lax-Oleinik formula. Also, because of the convergence of $\mathcal{T}(t) u_{0}$ on $\mathcal{M}_{0}$, it coincides with any element $\psi$ of $\omega\left(u_{0}\right)$ on $\mathcal{M}_{0}$. This is therefore the perfect candidate for convergence. To prove that it is indeed the goal of our quest, we need the last

Proposition 2. Let $u(t, x): \mathbb{R} \times \mathbf{T}^{n} \longrightarrow \mathbb{R}$ be a global and bounded viscosity solution of (3.1) such that $u=\phi$ on $\mathcal{M}_{0}$, then $u=\phi$ on $\mathbf{T}^{n}$.

The proof of this result is a simple extension of Theorem 2, and concludes the proof of Theorem 1 .

Particular cases. 1 . Let us come back to the hamiltonian $H(x, p)=|p|^{2}-f(x)$, where $f$ is nonnegative with nontrivial zero set - that we have already identified with $\mathcal{M}_{0}$. Here the convergence on $\mathcal{M}_{0}$ is not difficult to prove: for every $x \in \mathcal{M}_{0}$, the function $t \mapsto \mathcal{T}(t) u_{0}$ is nonincreasing. The convergence proof of $\mathcal{T}(t) u_{0}$ to a steady solution may then proceed by a Kruzhkov transform; see [26]. The idea of using the Kruzhkov transform - in a rather elaborate way - is also the basis of the purely PDE proof of Barles and Souganidis [8].

2. The case $n=1$. Here the dynamics of the extremals is known: they either converge to critical points of the hamiltonian, or cover the whole circle. In the latter case, the steady solutions are smooth. This fact was noted in [30], which may be considered as an embryo of the proof of Theorem 1 .

Remark. A striking fact of Theorem [ is that its proof does not necessitate any knowledge of the dynamics of the Lagrangian semigroup $S^{t}$, which might be extremely complicated. Unfortunately, this situation is not going to prevail in the non-strictly convex, or time-dependent case. The only examples - to this date - where one can say something about the large time dynamics of (1.1) are except the strictly convex, autonomous case - the ones where a good knowledge of the underlying Lagrangian dynamics is available.

\section{When convergence breaks down}

In this section we review two - very similar, as a matter of fact - cases where relatively complete information on the large-time dynamics of (1.1) is known: time-dependent Hamiltonians on the circle, and eikonal equations in the plane.

\subsection{Time-periodic hamiltonians}

This time, (1.1) becomes

$$
u_{t}+H\left(t, x, u_{x}\right)=0, \quad x \in \mathbf{T}
$$

where $\mathbf{T}:=\mathbf{T}^{1}$ is the unit circle. The Hamiltonian $H(t, x, p): \mathbb{R} \times \mathbf{T} \times \mathbb{R} \mapsto \mathbb{R}$ is $C^{2}$, 1-periodic in $t$, strictly convex in $p$, super-linear, and satisfies the following completeness assumption: the Hamiltonian vector-field

$$
X(t, x, p)=\left(H_{p}(t, x, p),-H_{x}(t, x, p)\right)
$$


is complete, i.e. for all $\left(t_{0}, x_{0} \cdot p_{0}\right)$, there exists a $C^{2}$ curve $\gamma(t)=(x(t), p(t)): \mathbb{R} \longrightarrow \mathbf{T} \times \mathbb{R}$ such that $\left(x\left(t_{0}\right), p\left(t_{0}\right)\right)=\left(x_{0}, p_{0}\right)$ and $\dot{\gamma}(t)=X(t, \gamma(t))$ for all $t \in \mathbb{R}$. Note that this hypothesis is satisfied if there exists a constant $C$ such that $\left|H_{t}\right| \leq C(1+H)$.

Once again, the Cauchy Problem for (3.1) is well posed in the viscosity sense: given a time $s \in \mathbb{R}$ and a continuous function $u_{0}: \mathbf{T} \rightarrow \mathbb{R}$, equation (3.1) has a unique viscosity solution $u(t, x)$ : $\left[s,+\infty\left[\times \mathbf{T} \longrightarrow \mathbb{R}\right.\right.$, such that $u(s,)=.u_{0}$. It will be denoted by $\mathcal{T}(s, t) u_{0}$. Also - and this is not specific to the one-dimensional setting, there exists, as in the autonomous setting, a real number $\lambda$ such that $u(t, x)-\lambda t$ is bounded for all viscosity solution $u:[s,+\infty[\times \mathbf{T} \longrightarrow \mathbb{R}$ of (3.1). Once again we may assume, without loss of generality, that $\lambda=0$.

The main result that we are going to comment on in this section is the following.

Theorem 3. ([29], [Q]], [10]) Let $u(t, x):[s,+\infty[\times \mathbf{T} \longrightarrow \mathbb{R}$ be a viscosity solution of (3.1]). There exist an integer $T$ and a T-periodic in $t$ viscosity solution: $\phi(t, x)$ such that

$$
\lim _{t \longrightarrow \infty}\|u(t, .)-\phi(t, .)\|_{\infty}=0
$$

Remarks. 1. Strictly speaking, convergence here does not break down, since we still recover some asymptotic time-periodicity. What breaks down anyway is the convergence to 1-periodic solutions in time: there are indeed viscosity solutions of equations of the form (3.1) which do not converge to 1-periodic solutions, see [8] and [20]. More precisely, one can build solutions which are periodic in time, but of minimal period greater than one.

2. The case $n>1$ is essentially open.

Let us denote $\mathcal{T}(s, t): C(\mathbf{T}, \mathbb{R}) \longrightarrow C(\mathbf{T}, \mathbb{R})$ the mapping which associates to each function $u_{0} \in C(\mathbf{T}, \mathbb{R})$ the function $u(t,$.$) , where u(t, x) \in C([s,+\infty[\times \mathbf{T}, \mathbb{R})$ is the viscosity solution of (3.1) such that $u(s,)=.u_{0}$.

We have the Markov property

$$
\mathcal{T}\left(t, t^{\prime}\right) \mathcal{T}(s, t)=\mathcal{T}\left(s, t^{\prime}\right)
$$

for $s \leq t \leq t^{\prime}$, hence the mappings $\mathcal{T}(0, n)=\mathcal{T}(0,1)^{n}, n \in \mathbb{N}$ form a discrete semi-group. We will note $\mathcal{T}$ for $\mathcal{T}(0,1)$ for simplicity. All the properties listed in Sections 2.1 are true, as well as the regularity results emanating from the Lax-Oleinik formula - which is of course also true here. Things begin to change when we look at the calibrated curves: one may not define a continuous dynamical system with them, only a discrete one. This rather innocent looking change causes a lot of trouble in Section 2.3.

More precisely, let $\phi(t, x)$ be a 1-periodic solution of (3.1). Mather's shortening lemma still implies that that two curves calibrated by $\phi$ cannnot intersect. Let

$$
\mathcal{D}^{\phi} \subset \mathbb{R} \times \mathbf{T}
$$

be the union of the graphs of these curves, and $\mathcal{D}_{0}^{\phi} \subset \mathbf{T}$ be the set of points $\gamma(0)$, where $\gamma: \mathbb{R} \longrightarrow \mathbf{T}$ is calibrated. This is a nonempty compact set. For each $t$, we define the mapping $S^{t}: \mathcal{D} \longrightarrow \mathbf{T}$ which associates to each $x \in \mathcal{D}_{0}$, the value $\gamma(t)$, where $\gamma: \mathbb{R} \longrightarrow \mathbf{T}$ is the unique calibrated curve satisfying $\gamma(0)=x$. It is a bi-Lipschitz homeomorphism onto its image. 
Clearly, $S^{1}$ is a homeomorphism of $\mathcal{D}_{0}$. Call it $S^{\phi}$ - to emphasise its dependence on $\phi$. Let us note $\mathcal{M}_{0}^{\phi}$ its $\omega$-limit. This is the closure in $\mathbf{T}$ the set of points $x \in \mathcal{D}_{0}^{\phi}$ which are the limit of a sequence $\left(S^{\phi}\right)^{n_{k}}(y)$ with $y \in \mathcal{D}_{0}$ and $n_{k}$ an increasing sequence of integers. The set $\mathcal{M}_{0}^{\phi}$ is nonempty and compact. As in the preceding section, the objects constructed in this section depend on the periodic solution $\phi$ that was chosen in the beginning.

Now, what is left from Section 2 are Lemma 1, Corollary 1 and Corollary \&. Suffice it to say that their non-autonomous analogues hold; we are not going to re-state them here. Consequently, if $\psi$ is another 1-periodic viscosity solution, Lemma 1 implies that the orbits of $\mathcal{M}_{\psi}$ are calibrated by $\phi$. It follows that the sets

$$
\mathcal{A}=\bigcap_{\phi} \mathcal{D}_{\phi}, \quad \mathcal{M}_{0}=\bigcap_{\phi} \mathcal{M}_{\phi}
$$

are not empty, where the intersection is taken on the set of 1-periodic viscosity solutions.

Let us denote by $\mathcal{T}$ the map $\mathcal{T}(0,1)$. As in Section 2 we have the

Theorem 4. $\mathcal{M}_{0}$ is a uniqueness set in the following sense: the Dirichlet problem with unknown $\phi:$

$$
\mathcal{T} \phi=\phi \text { on } \mathbf{T} \backslash \mathcal{M}_{0}, \quad \phi \text { imposed on } \mathcal{M}_{0}
$$

has at most one solution.

The problem is that, as opposed to Section 2, we do not have $\mathcal{T}(0, t) \mathcal{M}_{0}=\mathcal{M}_{0}$ for $t \in[0,1]$ - otherwise the question would be now settled. However, we may take advantage of the low dimension, and make use of Poincaré theory of homeomorphisms of the circle, see [22] for example. Recall that the map $S$ is a homeomorphism of the closed set $\mathcal{M}_{0}$; hence it has a well defined rotation number $\rho \in \mathbb{R}$. Recall that this rotation number can be defined by lifting $\mathcal{M}_{0}$ to the universal cover $\mathbb{R}$ of $\mathbf{T}$, as well as the map $S$ into a map $\tilde{S}$. We set

$$
\rho=\lim _{n \rightarrow+\infty} \frac{\tilde{S}^{n} x}{n}
$$

for any $x \in \mathcal{M}_{0}$. This number does not depend on the chosen point $x$, hence it only depends on the hamiltonian $H$.

Now, the proof of Theorem 3 goes as follows. Let $u(t, x)$ be an $\omega$-limit viscosity solution, and set

$$
\phi(t, x)=\liminf _{n \rightarrow+\infty} u(t+n, x)
$$

It all amouts to proving that $u=\phi$ in the two cases $\rho=0$ and $\rho$ irrational. Also, it is enough to prove equality on $\mathcal{M}_{0}$.

Case 1. $\rho=0$. In this case, $\mathcal{M}_{0}$ is a union of fixed points of $S^{1}$, and Lemma 1 finishes the proof. Notice that the case $\rho$ rational $\neq 0$ follows immediately: if $\rho=\frac{p}{q}$, with $p \wedge q=1$ the change of variable $x \mapsto x-\frac{p}{q} t$ brings us back to a $q$-time peridodic Hamiltonian whose global minimizing curves have zero rotation number.

Case 2. $\rho$ is irrational. This time one has to use Corollary \& in conjunction with the fact that there is a dense orbit of $S$ in $\mathcal{M}_{0}$. This last fact implies the uniqueness - up to the addition of constants of 1-periodic solutions; see [15], where this seems to have been first noticed. 


\subsection{Eikonal equations}

In the preceding section, we still had convergence respecting the structure and periodicity of the hamiltonian, but this is because strict convexity was still there. We are going to see in this section that the absence of strict convexity can have dramatic effets, and an instance is not to be sought for very far: consider the equation

$$
u_{t}+\left|u_{x}+1\right|=1, \quad u(0, x)=\sin x
$$

It is readily seen that the solution is $u(t, x)=\sin (x-t)$, which has no chance to converge to anything steady. This beautiful example is due to Barles and Souganidis [7].

We want here to discuss some 2D generalisations, and explain why - at least in the $2 \mathrm{D}$ case - we may end up with time-periodic solutions. Our motivation here is the large time behaviour of 2D fronts propagating under eikonal equations, namely

$$
V_{n}=R(X)
$$

where $V_{n}$ is the normal speed of the front at the point $X$ and $R: \mathbb{R}^{N} \rightarrow R$ is a smooth, $\mathbb{Z}^{N}$-periodic function which is controlled from below by a positive constant. The underlying physics is a model of solid combustion in heterogeneous media where the flame front is assumed to propagate in $\mathbb{R}^{N}$ with a periodic, space dependent normal velocity. Although extremely simple, this phenomenological model gives useful information, due to the lack of a definite burning rate theory in solid combustion. This type of models was already proposed by Landau in the 40's, in particular a simpler example when $R$ is a constant: a weak formulation to study the simple Landau model was proposed in Barles [2]; it was based on the idea that the moving front can be identified as the 0-level set of the unique viscosity solution of an eikonal equation. This idea was first used for numerical computations by Osher and Sethian [27] who extended it to more general normal velocities (in particular curvature dependent ones). The theoretical basis of the so-called "level set approach" was set by Evans and Spruck [16], Chen, Giga and Goto [13].

In our case, the level set approach for (3.2) consists in solving the eikonal equation

$$
u_{t}+R(x)|D u|=0 \quad \text { in }(0,+\infty) \times \mathbb{R}^{N},
$$

the flame front at time $t$, denoted by $\Gamma_{t}$, being identified as the 0-level set of $u(t, \cdot)$.

Equation (3.3) generates a nonlinear semigroup denoted by $\mathcal{T}(t)$, and the solution of the Cauchy problem for (3.3) is given by the following (completely degenerate, in view of the LaxOleinik formula) explicit formula:

$$
u(t, x)=\inf _{\gamma(t)=x,|\dot{\gamma}| \leq R(\gamma)} u(0, \gamma(0))
$$

where $\gamma$ is taken among all piecewise $C^{1}$ curves.

Now, consider a vector $p \in \mathbb{Z}^{2}$; in order to model a motion whose general direction of propagation is the vector $p$, we impose an initial data $u_{0}$ to (3.3) which is of the form

$$
u_{0}(x)=p \cdot x+v_{0}(x)
$$


where $v_{0}$ is a bounded, continuous, $\mathbb{Z}^{2}$-periodic function. The corresponding solution $u(t, x)$ of (3.3) has then the form

$$
u(t, x)=p \cdot x-\bar{H}(p) t+v(t, x),
$$

where $\bar{H}(p)$ is the constant $\lambda$ of Section 2. The function $v$ is $\mathbb{Z}^{2}$-periodic in $x$ and solves

$$
\begin{aligned}
v_{t}+R(x)|D v+p| & =\bar{H}(p) & & \text { in }(0, \infty) \times \mathbb{R}^{2} \\
v(0, x) & =v_{0}(x) & & \text { in } \mathbb{R}^{2} .
\end{aligned}
$$

Once again, Equation (3.5) generates a nonlinear, weakly contracting semigroup that is still denoted by $\mathcal{T}(t)$, in the space of $\mathbb{Z}^{2}$ periodic functions. Notice that (3.4) does not give any compactness, but an initially Lipschitz solution will remain uniformly Lipschitz - this is easily seen by first bounding $u_{t}$, then $D u$. Hence we may take $\omega$-limit sets in $C\left(\mathbf{T}^{2}\right)$. Choose a steady $\mathbb{Z}^{2}$-periodic solution $\phi$ (3.5); we may trap $v_{0}$ between $\phi-C$ and $\phi+C$, where $C>0$ is a large enough constant: the maximum principle implies

$$
\forall t>0, \quad \phi(x)-C \leq v(t, x) \leq \phi(x)+C .
$$

This last inequality already gives a nontrivial information, i.e: the asymptotic velocity of the front is $\bar{H}(p)$; notice that it is valid in any space dimension. More specific to space dimension 2 is the

Theorem 5. (/[j]) There exists $T(p)>0$ such that, for every $v_{0} \in \mathcal{C}_{p e r}$, there is a function $v_{\infty}(t, x)$, which is $T(p)$ periodic in time, and such that

$$
\lim _{t \rightarrow+\infty}\left\|\mathcal{T}(t) v_{0}-v_{\infty}(t, .)\right\|_{\infty}=0
$$

Outline of the proof. A steady solution of (3.5) is a steady solution of

$$
H(x, D \phi):=R^{2}(x)|D \phi+p|^{2}-\bar{H}(p)^{2},
$$

and the hamiltonian $H$ is strictly convex. Therefore an Aubry-Mather $\mathcal{M}_{0}$ set can be defined as in Section 2, and it is sufficient to study $\mathcal{T}(t) v_{0}$ on $\mathcal{M}_{0}$.

Next, we have a monotonicity formula similar to Lemma it:

Lemma 2. Let $\gamma: \mathbb{R} \rightarrow \mathcal{M}_{0}$ be calibrated by $\phi$. Set

$$
\tilde{\gamma}(t)=\gamma\left(\frac{t}{2 \bar{H}(p)}\right) .
$$

Then the function

$$
t \mapsto v(t, \tilde{\gamma}(t))-\phi(\tilde{\gamma}(t))
$$

is nonincreasing on $\mathbb{R}_{+}$.

This lemma - and Corollary 1 - is essentially what survives from Sections 2.1, 2.2 and 2.3: strict convexity is missing here. The only way we have to replace it, is a better knowledge of the dynamics of the extremals, which is available in 2-D: see Bangert [1].

Remarks. 1. Lemma 2 exactly accounts for whan happens in the Barles-Souganidis example: the dynamics of the globally calibrated curves of $\mathcal{M}_{0}$ dictates the dynamics of $\mathcal{T}(t) v_{0}$. in this particular 
$1 D$ example, $\mathcal{M}_{0}$ is the whole circle, but is also made up of one orbit; the fact that it travels at unit speed is then reflected in the periodicity of the initial datum, which eventually produces the observed time-periodicity.

2. A nontrivial example can be treated as follows: assume the existence of a strip - say, $\left[-\frac{1}{4}, \frac{1}{4}\right] \times \mathbb{R}$ where the funtion $R$ is very large - say, $10^{4}$ times larger than in $\left(\left[-\frac{1}{2}, \frac{1}{2}\right] \backslash\left[-\frac{3}{8}, \frac{3}{8}\right]\right) \times \mathbb{R}$. The orbits of $\mathcal{M}_{0}$ have zero rotation number, hence are 1-periodic. The function $\mathcal{T}(t) v_{0}$ becomes asymptotically periodic of period $2 \bar{H}(p)$.

Needless to say, the problem is once again essentially open in 3 space dimensions.

\section{Unbounded solutions}

A natural question is to ask what is left from Section 2 if the periodicity assumption is removed. But then there is no real reason why (1.4) should have bounded solution for any kind of $\lambda$. The correct place where to look for solutions is then the space of Lipschitz solutions, as easily seen on the trivial example

$$
u_{t}+u_{x}^{2}=0, \quad x \in \mathbb{R} .
$$

It is seen by inspection that (1.4) has Lipschitz continuous - but unbounded - solutions as soon as $\lambda \geq 0$; the particular case of $\lambda=0$ yielding solutions that are additionally bounded. This phenomenon is general, and our starting point is to find the correct analogue of the Lions-PapanicolaouVaradhan Lemma. In this section, we will be interested in the Hamilton-Jacobi equation

$$
u_{t}+H(x, D u)=0 ; \quad x \in \mathbb{R}^{n}
$$

and the corresponding ergodic problem is

$$
H(x, D u)=\lambda ; \quad x \in \mathbb{R}^{n} .
$$

Proposition 3. ([6], [19]) Assume that $H$ is bounded, uniformly continuous on $\mathbb{R}^{n} \times B(0, R)$ for any $R>0$; and assume that it is coercive. Then there exists $\lambda_{\text {min }} \in \mathbb{R}$ such that for any $\lambda \geq \lambda_{\text {min }}$, there exists a Lipschitz continuous solution of (4.2).

We notice that there is no need of any kind of convexity in this result, which can be proved by a standard approximation argument.

The trouble is that now, there are a lot of ergodic constants; hence a solution of 4.1 need not be ergodic anymore. This fear is confirmed by the following construction, detailed in [5]: take $n=1$, and $H(x, p)=p^{2}$. Let $\left(a_{n}\right)_{n \in \mathbb{N}}$ be a strictly increasing sequence of non-negative real numbers such that

$$
\lim _{n} \frac{a_{n+1}}{a_{n}}=+\infty .
$$

We consider the Lipschitz continuous initial data $u_{0}$ defined in the following way

$$
u_{0}(y)=0 \text { for } y \leq a_{0},
$$

and for any $k \in \mathbb{N}$

$$
u_{0}^{\prime}(y)= \begin{cases}0 & \text { if } y \in\left(a_{2 k+1}, a_{2 k+2}\right) \\ -1 & \text { if } y \in\left(a_{2 k}, a_{2 k}\right)\end{cases}
$$

It then occurs that 
Proposition 4. There are two sequences increasing to $+\infty:\left(t_{n}\right)_{n}$ and $\left(t_{n}^{\prime}\right)_{n}$ such that

$$
\lim _{n \rightarrow+\infty} \frac{u\left(t_{n}, 0\right)}{t_{n}}=0, \quad \lim _{n \rightarrow+\infty} \frac{u\left(t_{n}^{\prime}, 0\right)}{t_{n}^{\prime}}=\frac{3}{2} .
$$

A very interesting open problem is to provide a general description of this loss of ergodicity. We believe that a mixing phenomenon occurs, as for instance in Hamel-Nadirashvili [21] for the FisherKPP equation. As a consequence the theory in that case is far from complete, and some progress is to be expected. In particular, uniqueness conditions for (4.2) are far from being clear, and it would be desirable to investigate this question, before significant progress for the general theory of (4.2) is made.

An perhaps more accessible question is: what set of reasonable assumptions can guarantee convergence? Here are two results, taken from [6].

Theorem 6. Assume that the assumptions of Proposition $⿴$ 年old and that $H$ is strictly convex in $p$. If the initial data $u_{0}$ satisfies

$$
\lim _{|x| \rightarrow+\infty}\left(u_{0}(x)-\phi(x)\right)=0,
$$

where $\phi: \mathbb{R}^{n} \rightarrow \mathbb{R}$ is a solution of (4.2) for some $\lambda>\lambda_{\text {min }}$, then we have

$$
u(x, t)+\lambda t \rightarrow \phi(x) \quad \text { locally uniformly in } \mathbb{R}^{n} \text { as } t \rightarrow+\infty .
$$

The proof relies on the fact that the curves $\gamma_{t}$ calibrated by $u$ on the interval $[0, t]$ satisfy

$$
\lim _{t \rightarrow+\infty}\left|\gamma_{t}(0)\right|=+\infty
$$

in obvious contrast with what happens in the periodic case - at least with Hamiltonians of the form $p^{2}-f(x)$. It would be very interesting to try to relax (4.3).

The next and final result is a Liouville type theorem, about time-global solutions of (4.1).

Theorem 7. Assume the assumptions of Proposition $\square$ hold and that $H$ is strictly convex in $p$. Consider $u(t, x)$ a time-global - i.e. defined for all $t \in \mathbb{R}$ - solution of (4.1), for which there exists $\lambda \geq \lambda_{\text {min }}$ and a solution $\phi$ of (4.2) such that $(t, x) \mapsto u(t, x)-\lambda t-\phi(x)$ is bounded on $\mathbb{R} \times \mathbb{R}^{n}$.

Then there is a constant $C \in \mathbb{R}$ such that $u(t, x)=\lambda t+\phi(x)+C$.

The proof relies on PDE arguments of the type [7]. We do not know a dynamical systems proof of it.

Once again, it would be interesting to find conditions close to necessary and sufficient such that Theorem 7 holds.

\section{References}

[1] V. BANGERT, Mather sets for twist maps and geodesics on tori, Dynamics reported, 1, pp. 1-56, Dynam. Reported, Ser. Dynam. Systems Appl., Wiley, Chichester, 1988.

[2] G. BARLES, Remark on a flame propagation model, Rapport INRIA, 464 (1985). 
[3] G. BArles, Solutions de viscosité des ÉQuations de Hamilton-Jacobi. Collection "Mathématiques etApplications" of SMAI, n¹7, (1994), Springer-Verlag.

[4] G. Barles, B. Perthame, Discontinuous solutions of deterministic optimal stopping time problems, M2AN, 21 (1987), 557-579.

[5] G. BARLES, J.-M. ROQUEJOFFRE, Large time behaviour of fronts governed by eikonal equations, Interfaces Free Bound. 5 (2003), pp. 83-102.

[6] G. BARLES, J.-M. RoQUEJOFFRE, Ergodic type problems and large time behavior of solutions of Hamilton-Jacobi Equations in an unbounded framework, Comm. Partial Diff. Eq., to appear.

[7] G. BARLES, P.E. SougAnidis, On the large time behaviour of solutions of Hamilton-Jacobi equations, SIAM J. Math. Anal. 31, 4 (2000), 925-939.

[8] G. BARLES, P.E. SougANIDIS, Some counterexamples on the asymptotic behaviour of the solutions of Hamilton-Jacobi equations, C. R. Acad. Sci., Paris, Série I, 330, (2000), 963-968.

[9] P. Bernard, Connecting orbits of time dependent Lagrangian systems. Ann. Inst. Fourier 52 (2002), 1533-1568.

[10] P. Bernard, J.-M. Roquejoffre, Convergence to Time-Periodic Solutions in Time-Periodic Hamilton-Jacobi Equations on the Circle, Comm. Partial Diff. Eq., 29 (2004), pp. 457 - 469.

[11] L.A. Caffarelli, R. DE la Llave, Planelike minimizers in periodic media, Comm. Pure Appl. Math. 54 (2001), pp. 1403-1441.

[12] L.A. Caffarelli, P.E. Souganidis, L. WAng, Homogenisation of fully nonlinear, uniformly elliptic and parabolic partial differential equations in stationary ergodic media, Comm. Pure Appl. Math., 58 (2005), pp. p 319-361.

[13] Y.-G. ChEn, Y. GIGA AND S. Goto, Uniqueness and existence of viscosity solutions of generalized mean curvature flow equations, J. Diff. Geom. 33 (1991), 749-786.

[14] A. DAVini, A. SiCONOLFI, A generalised dynamical approach to the large time behaviour of solutions of Hamilton-Jacobi equations, preprint.

[15] W. E, Aubry-Mather theory and periodic solutions of the forced Burgers equation, Comm. Pure Appl. Math. 52 (1999), 811-828.

[16] L. C. Evans, J. Spruck, Motion of level sets by mean curvature I, J. Diff. Geom. 33 (1991), 635-681.

[17] A. FATHI, Théorème KAM faible et théorie de Mather sur les systèmes lagrangiens, C. R. Acad. Sci. Paris, Série I, 324 (1997), 1043-1046.

[18] A. FATHI, Sur la convergence du semi-groupe de Lax-Oleinik. C. R. Acad. Sci., Paris, Série I, 327,(1998), 267-270.

[19] A. FATHI, E. MADERnA, Weak KAM theorem on non compact manifolds, to appear in NoDEA.

[20] A. FATHI, J.N. MATHER, Failure of convergence of the Lax-Oleinik semi-group in the time-dependent case, Bull. Soc. Math. France, 128 (2000), 473-483.

[21] F. HAmel, N. NAdiRAshVILI, Travelling waves and entire solutions of the Fisher-KPP equation in $\mathbb{R}^{N}$, Arch. Ration. Mech. Anal. 157 (2001), pp. 91-163.

[22] A. KATOK, B. HASSELBLATT, Introduction to the modern theory of dynamical systems. Encyclopedia of Mathematics and its Applications, 54. Cambridge University Press, Cambridge, (1995). 
[23] J. N. MATHER, Action minimizing invariant measures for positive defi nite Lagrangian systems, Math. Z. 207, 169-207 (1991)

[24] P.-L. Lions, Generalized solutions of Hamilton-Jacobi Equations, Research Notes in Mathematics, Pitman.

[25] P.-L. Lions, G. PAPAnicolaou, S.R.S VARAdhan, Homogenization of Hamilton-Jacobi equations; unpublished preprint.

[26] G. NAmAh, J.-M. RoQUeJOFFRE, Remarks on the long time behaviour of the solutions of Hamilton-Jacobi Equations, Commun. Partial Differ. Equations 24 (1999), pp. 883-893 .

[27] S. Osher, J. Sethian, Fronts moving with curvature dependent speed: algorithms based on Hamilton-Jacobi equations, J. Comp. Phys. 79 (1988), 12-49.

[28] M. Pierre, E. Rouy, A tridimensional inverse shaping problem, Comm. Partial Diff. Eq., 21 (1996), pp. 1279-1305.

[29] J.-M. RoQUEJOFFRE, Convergence to steady states or periodic solutions in a class of Hamilton-Jacobi equations, J. Math. Pures Appl., 80 (2001), pp. 85-104.

[30] J.-M. RoQUeJoffre, Comportement asymptotique des solutions d'équations de Hamilton-Jacobi monodimensionnelles, C. R. Acad. Sci. Paris, 326, Série I, pp. 185-189 (1998). 